\title{
A Note on Interior Bases of Semigroups
}

\author{
WALUNYAPORN PANATE, WICHAPON CHATTHONG, WARUD NAKKHASEN* \\ Department of Mathematics, Faculty of Science, Mahasarakham University \\ Maha Sarakham, 44150 THAILAND
}

\begin{abstract}
The concept of two-sided bases of a semigroup $S$ was introduced by Fabrici in 2009. In this paper, we introduce the concept of interior bases of a semigroup $S$ which is based on the result of interior ideals generated by a nonempty subset of the semigroup $S$. Then, we study some results of a semigroup $S$ containing interior bases and characterize when a nonempty subset of a semigroup $S$ is an interior base of $S$.
\end{abstract}

Key-Words: - semigroup, interior ideal, interior base, quasi-order

Received: February 10, 2021. Revised: August 3, 2021. Accepted: September 1, 2021. Published: September 20, 2021.

\section{Introduction and Preliminaries}

The concepts of left bases and right bases of a semigroup were introduced by Tamura [1]. Later, Fabrici [2] introduced and studied the structure of a semigroup containing one-sided bases, namely, two-sided bases.

Definition 1.1. [2] $A$ subset $A$ of a semigroup $S$ is called a two-sided base of $S$ if it satisfies the following conditions:

(i) $S=A \cup S A \cup A S \cup S A S$;

(ii) if $B$ is a subset of $A$ such that $S=B \cup S B \cup$ $B S \cup S B S$, then $B=A$.

When a nonempty subset of a semigroup $S$ is a two-sided base of $S$, the author provided a characterization. Afterward, Fabrici and Kepka proved in [3] that there is a relationship between a semigroup's bases and maximal ideals.

Changphas and Sammaprab [4] have extended the conclusions obtained by Fabrici [2] to ordered semigroups. Next, based on the result of bi-ideals generated by a nonempty subset of a semigroup $S$ called bibases, was introduced by Kummoon and Changphas [5]. In 2021, Jantanan, Budpan and Loesna [6] studied some properties of ordered $\Gamma$-semihypergroups containing two-sided bases. Recently, the concept of bi-bases was introduced and discussed in semihypergroups and ordered $\Gamma$-semihypergroups by [7] and [8], respectively.

In 1976, Lajos [9] gave the concept of interior ideals of semigroups.

Definition 1.2. [9] A subsemigroup I of a semigroup $S$ is called an interior ideal of $S$ if $S I S \subseteq I$.
This concept generalizes the concept of two-sided ideals in semigroups. Then, the concept of interior ideals has been studied in other algebraic structures, for example, [10, 11, 12, 13, 14, 15].

Let $\left\{I_{i} \mid i \in \Lambda\right\}$ be a family of interior ideals of a semigroup $S$. It is known that if $\bigcap_{i \in \Lambda} I_{i} \neq \emptyset$, then $\bigcap I_{i}$ is also an interior ideal of $S$. Furthermore, for $i \in \Lambda$ any nonempty subset $A$ of $S$, we denote $(A)_{I}$ as the smallest interior ideal of $S$ containing $A$. For any $a \in$ $S$, we denote $(a)_{I}=(\{a\})_{I}$. The form of $(A)_{I}$ is shown in the following lemma.

Lemma 1.3. If $A$ is a nonempty subset of a semigroup $S$, then $(A)_{I}=A \cup A A \cup S A S$.

Proof. Put $N=A \cup A A \cup S A S$. Obviously, $A \subseteq N$. Next, we consider

$$
\begin{aligned}
N N & =(A \cup A A \cup S A S)(A \cup A A \cup S A S) \\
& \subseteq A A \cup S A S \\
& \subseteq A \cup A A \cup S A S=N
\end{aligned}
$$

and

$$
\begin{aligned}
S N S & =S(A \cup A A \cup S A S) S \\
& \subseteq S A S \subseteq A \cup A A \cup S A S=N .
\end{aligned}
$$

Hence, $N$ is an interior ideal of $S$. Let $K$ be any interior ideal of $S$ containing $A$. It follows that $N=$ $A \cup A A \cup S A S \subseteq K \cup K K \cup S K S \subseteq K$. Therefore, $N$ is the smallest interior ideal of $S$ containing $A$, that is, $(A)_{I}=N=A \cup A A \cup S A S$.

In a particular case of Lemma 1.3, if $A=\{a\}$ then we have the following corollary. 
Corollary 1.4. If $S$ is a semigroup and $a \in S$, then $(a)_{I}=a \cup a a \cup S a S$.

Since the interior ideal is a kind of ideals that is popularly studied in semigroups, then it is important to investigate based on the result of interior ideals of semigroups. The purpose of this paper to introduce the concept of interior bases in a semigroup $S$ which derive from interior ideals generated by a nonempty subset of the semigroup $S$. Then, we discuss the structure of a semigroup $S$ containing interior bases.

\section{Main Results}

In this section, we give the definition of an interior base of a semigroup $S$ and characterize when a nonempty subset of $S$ is an interior base by using the quasi-order on $S$ defined by the principal interior ideals of $S$.

Definition 2.1. Let $S$ be a semigroup. A subset $A$ of $S$ is called an interior base of $S$ if it satisfies the following two conditions:

(i) $S=(A)_{I}$ (i.e., $S=A \cup A A \cup S A S$ );

(ii) if $B$ is a subset of $A$ such that $S=(B)_{I}$, then $A=B$.

Example 2.2. Let $S=\{a, b, c, d\}$ be a semigroup with the binary operation . on $S$ defined by the following table:

\begin{tabular}{c|cccc}
$\cdot$ & $a$ & $b$ & $c$ & $d$ \\
\hline$a$ & $a$ & $a$ & $a$ & $a$ \\
$b$ & $a$ & $a$ & $a$ & $a$ \\
$c$ & $a$ & $a$ & $b$ & $a$ \\
$d$ & $a$ & $a$ & $b$ & $b$
\end{tabular}

By routine calculations, we obtain that $\{c, d\}$ is an interior base of $S$.

Example 2.3. Consider $S=\{a, b, c, d\}$ is a semigroup with the multiplication on $S$ defined by:

\begin{tabular}{c|cccc}
$\cdot$ & $a$ & $b$ & $c$ & $d$ \\
\hline$a$ & $a$ & $b$ & $a$ & $a$ \\
$b$ & $b$ & $a$ & $b$ & $b$ \\
$c$ & $a$ & $b$ & $c$ & $d$ \\
$d$ & $a$ & $b$ & $d$ & $c$
\end{tabular}

Let $A=\{c, d\}$. We have that $S=A \cup A A \cup S A S$, but $A$ is not an interior base of $S$ because there exists a subset $\{c\}$ of A such that $S=\{c\} \cup\{c\}\{c\} \cup S\{c\} S$, and importantly, $\{c\} \neq A$. Moreover, we can show that $\{c\}$ and $\{d\}$ are interior bases of $S$.

Lemma 2.4. Let I be an interior base of a semigroup $S$ and $a, b \in I$. If $a \in b b \cup S b S$, then $a=b$.
Proof. Assume that $a \in b b \cup S b S$, and suppose that $a \neq b$. Put $A:=I \backslash\{a\}$. Thus, $A \subset I$. Since $a \neq b$, $b \in A$. Next, we will show that $(A)_{I}=S$. Clearly, $(A)_{I} \subseteq S$. Let $x \in S$. Then, by $(I)_{I}=S$, it follows that $x \in I \cup I I \cup S I S$. So, there are three cases to consider:

Case 1: $x \in I$.

Subcase 1.1: $x \neq a$. Then,

$$
x \in I \backslash\{a\}=A \subseteq(A)_{I} .
$$

Subcase 1.2: $x=a$. By assumption,

$$
x=a \in b b \cup S b S \subseteq A A \cup S A S \subseteq(A)_{I} .
$$

Case 2: $x \in I I$. Then, $x=b_{1} b_{2}$ for some $b_{1}, b_{2} \in I$.

Subcase 2.1: $b_{1}=a$ and $b_{2}=a$. By assumption,

$$
\begin{aligned}
x=b_{1} b_{1} & \in(b b \cup S b S)(b b \cup S b S) \\
& =b b b b \cup b b S b S \cup S b S b b \cup S b S S b S \\
& \subseteq A A A A \cup A A S A S \cup S A S A A \cup S A S S A S \\
& \subseteq S A S \subseteq(A)_{I} .
\end{aligned}
$$

Subcase 2.2: $b_{1} \neq a$ and $b_{2}=a$. Then, $b_{1} \in$ $I \backslash\{a\}=A$. By assumption,

$$
\begin{aligned}
x=b_{1} b_{1} & \in A(b b \cup S b S) \\
& =A b b \cup A S b S \\
& \subseteq A A A \cup A S A S \\
& \subseteq S A S \subseteq(A)_{I} .
\end{aligned}
$$

Subcase 2.3: $b_{1}=a$ and $b_{2} \neq a$. Then, $b_{2} \in A$. By assumption,

$$
\begin{aligned}
x=b_{1} b_{1} & \in(b b \cup S b S) A \\
& =b b A \cup S b S A \\
& \subseteq A A A \cup S A S A \\
& \subseteq S A S \subseteq(A)_{I} .
\end{aligned}
$$

Subcase 2.4: $b_{1} \neq a$ and $b_{2} \neq a$. Then, $b_{1}, b_{2} \in$ $I \backslash\{a\}=A$. It follows that,

$$
x=b_{1} b_{1} \in A A \subseteq(A)_{I} .
$$

Case 3: $x \in S I S$. Then, $x=s b_{3} t$ for some $b_{3} \in I$ and $s, t \in S$.

Subcase 3.1: $b_{3} \neq a$. Then, $b_{3} \in I \backslash\{a\}=A$. It turns out that

$$
x=s b_{3} t \in S A S \subseteq(A)_{I} .
$$

Subcase 3.2: $b_{3}=a$. By assumption,

$$
\begin{aligned}
x=s b_{3} t & \in s(b b \cup S b S) t \\
& \subseteq S A A S \cup S S A S S \\
& \subseteq S A S \subseteq(A)_{I} .
\end{aligned}
$$

This implies that $(A)_{I}=S$. This is a contradiction. Therefore, $a=b$. 
Lemma 2.5. Let I be an interior base of a semigroup $S$ and $a, b, c \in I$. If $a \in b c \cup S b c S$, then $a=b$ or $a=c$.

Proof. Assume that $a \in b c \cup S b c S$, and suppose that $a \neq b$ and $a \neq c$. Setting $A:=I \backslash\{a\}$. Then, $A \subset I$. Since $a \neq b$ and $a \neq c$, we have $b, c \in A$. We will show that $(A)_{I}=S$. Clearly, $(A)_{I} \subseteq S$. On the other hand, let $x \in S$, By $(I)_{I}=S, x \in I \cup I I \cup S I S$. We consider three cases:

Case 1: $x \in I$.

Subcase 1.1: $x \neq a$. Then,

$$
x \in I \backslash\{a\}=A \subseteq(A)_{I} .
$$

Subcase 1.2: $x=a$. By assumption,

$$
\begin{aligned}
x=a & \in b c \cup S b c S \\
& \subseteq A A \cup S A A S \\
& \subseteq A A \cup S A S \subseteq(A)_{I} .
\end{aligned}
$$

Case 2: $x \in I I$. Then, $x=b_{1} b_{2}$ for some $b_{1}, b_{2} \in I$.

Subcase 2.1: $b_{1}=a$ and $b_{2}=a$. By assumption,

$$
\begin{aligned}
x=b_{1} b_{2} \in & (b c \cup S b c S)(b c \cup S b c S) \\
= & b c b c \cup b c S b c S \cup S b c S b c \cup S b c S S b c S \\
\subseteq & A A A A \cup A A S A A S \cup S A A S A A \\
& \cup S A A S S A A S \\
\subseteq & S A S \subseteq(A)_{I} .
\end{aligned}
$$

Subcase 2.2: $b_{1} \neq a$ and $b_{2}=a$. Then, $b_{1} \in$ $I \backslash\{a\}=A$. By assumption,

$$
\begin{aligned}
x=b_{1} b_{2} & \in A(b c \cup S b c S) \\
& =A b c \cup A S b c S \\
& \subseteq A A A \cup A S A A S \\
& \subseteq S A S \subseteq(A)_{I} .
\end{aligned}
$$

Subcase 2.3: $b_{1}=a$ and $b_{2} \neq a$. Then, $b_{2} \in$ $I \backslash\{a\}=A$. By assumption,

$$
\begin{aligned}
x=b_{1} b_{2} & \in(b c \cup S b c S) A \\
& =b c A \cup S b c S A \\
& \subseteq A A A \cup S A A S A \\
& \subseteq S A S \subseteq(A)_{I} .
\end{aligned}
$$

Subcase 2.4: $b_{1} \neq a$ and $b_{2} \neq a$. Then $b_{1}, b_{2} \in$ $I \backslash\{a\}=A$. Thus,

$$
x=b_{1} b_{2} \in A A \subseteq(A)_{I} .
$$

Case 3: $x \in S I S$. Then, $x=s b_{3} t$ for some $b_{3} \in I$ and $s, t \in S$. Thus,

Subcase 3.1: $b_{3} \neq a$. Then, $b_{3} \in I \backslash\{a\}=A$.

$$
x=s b_{3} t \in S A S \subseteq(A)_{I} .
$$

Subcase 3.2: $b_{3}=a$. By assumption,

$$
\begin{aligned}
x=s b_{3} t & \in s(b b \cup S b S) t \\
& \subseteq S A A S \cup S S A S S \\
& \subseteq S A S \subseteq(A)_{I} .
\end{aligned}
$$

This implies that $(A)_{I}=S$. This is a contradiction. Therefore, $a=b$ or $a=c$.

Now, we need the quasi-order defined as follows to provide a characterization when a nonempty subset of a semigroup is interior base of the semigroup.

Definition 2.6. Let $S$ be a semigroup. Define a quasiorder on $S$ by for every $a, b \in S$,

$$
a \leqslant b: \Leftrightarrow(a)_{I} \subseteq(b)_{I} .
$$

Note that the relation $\leqslant$ defined above need not to be a partial order as shown by the following example.

Example 2.7. From Example 2.3, we see that $(c)_{I} \subseteq$ $(d)_{I}$ (i.e., $\left.c \leqslant d\right)$ and $(d)_{I} \subseteq(c)_{I}$ (i.e., $d \leqslant c$ ), but $c \neq d$. Hence, $\leqslant$ is not a partial order on $S$.

Lemma 2.8. Let I be an interior base of a semigroup $S$. If $a, b \in I$ such that $a \neq b$, then neither $a \leqslant b$, nor $b \leqslant a$.

Proof. Assume that $a, b \in I$ such that $a \neq b$. Suppose that $a \leqslant b$. Then, $a \in(a)_{I} \subseteq(b)_{I}$. Since $a \neq b$, we have $a \in b b \cup S b S$. By Lemma 2.4, $a=b$. This is a contradiction. For the case $b \leqslant a$, we can be proved similarly.

Lemma 2.9. Let I be a interior base of a semigroup $S$. For every $a, b, c \in I$ and every $s, t \in S$, then the following statements hold:

(i) if $a \in b c \cup b c b c \cup S b c S$, then $a=b$ or $a=c$;

(ii) if $a \in s b t \cup s b t s b t \cup S s b t S$, then $a=b$.

Proof. (i) Assume that $a \in b c \cup b c b c \cup S b c S$. Suppose that $a \neq b$ and $a \neq c$. Let $A:=I \backslash\{a\}$. Then, $A \subset I$. Since $a \neq b$ and $a \neq c$, we have that $b, c \in A$. We will show that $(I)_{I} \subseteq(A)_{I}$. It is sufficient to prove that $I \subseteq(A)_{I}$. Let $x \in I$. If $x \neq a$, then $x \in A$, and so $x \in(A)_{I}$. If $x=a$, then by given assumption, we have

$$
\begin{aligned}
x=a & \in b c \cup b c b c \cup S b c S \\
& \subseteq A A \cup A A A A \cup S A A S \\
& \subseteq A A \cup S A S \subseteq(A)_{I} .
\end{aligned}
$$

Hence, $I \subseteq(A)_{I}$. This implies that $(I)_{I} \subseteq(A)_{I}$. Since $I$ is an interior base of $S, S=(I)_{I} \subseteq(A)_{I} \subseteq$ $S$. Thus, $S=(A)_{I}$, which is a contradiction. Therefore, $a=b$ or $a=c$. 
(ii) Assume that $a \in s b t \cup s b t s b t \cup S s b t S$, and suppose that $a \neq b$. Letting $A:=I \backslash\{a\}$. Then, $A \subset I$. Since $a \neq b, b \in A$. We need to show that $I \subseteq(A)_{I}$. Let $x \in I$. If $x \neq a$, then $x \in A$, and so $x \in(A)_{I}$. If $x=a$, then by given assumption, we have

$$
\begin{aligned}
x=a & \in s b t \cup s b t s b t \cup S s b t S \\
& \subseteq S A S \cup S A S S A S \cup S S A S S \\
& \subseteq S A S \subseteq(A)_{I} .
\end{aligned}
$$

Thus, $I \subseteq(A)_{I}$, implies that $(I)_{I} \subseteq(A)_{I}$. Since $I$ is an interior base of $S, S=(I)_{I} \subseteq(A)_{I} \subseteq S$. Hence, $S=(A)_{I}$. This is a contradiction. Therefore, $a=b$.

Lemma 2.10. Let I be an interior base of a semigroup $S$. Then the following statements hold:

(i) for every $a, b, c \in I$, if $a \neq b$ and $a \neq c$, then $a \nless b c$;

(ii) for every $a, b \in I$ and every $s, t \in S$, if $a \neq b$, then $a \nless$ sbt.

Proof. (i) Let $a, b, c \in I$ such that $a \neq b$ and $a \neq c$. Suppose that $a \leqslant b c$. Then,

$$
a \in(a)_{I} \subseteq(b c)_{I}=b c \cup b c b c \cup S b c S .
$$

By Lemma 2.9(i), we have that $a=b$ or $a=c$. This is a contradiction to the assumption. It follows that $a \nless b c$.

(ii) Let $a, b \in I$ and $s, t \in S$ such that $a \neq b$. Suppose that $a \leqslant s b t$. We obtain that

$$
a \in(a)_{I} \subseteq(s b t)_{I}=s b t \cup s b t s b t \cup S s b t S .
$$

By Lemma 2.9(ii), we get that $a=b$, which is a contradiction to the assumption. Therefore, $a \nless s b t$.

Finally, we present the main result of this paper by characterizing when a nonempty subset of a semigroup $S$ is an interior base of $S$.

Theorem 2.11. Let I be a nonempty subset of a semigroup $S$. Then I is an interior base of $S$ if and only if I satisfies the following conditions:

(i) for every $x \in S$,

(i.a) there exists $a \in I$ such that $x \leqslant a$; or

(i.b) there exist $a_{1}, a_{2} \in I$ such that $x \leqslant a_{1} a_{2}$; or

(i.c) there exists $a_{3} \in I$ and there exist $s, t \in S$ such that $x \leqslant s a_{3} t$;

(ii) for every $a, b, c \in I$, if $a \neq b$ and $a \neq c$, then $a \nless b c$; (iii) for every $a, b \in S$ and every $s, t \in S$, if $a \neq b$, then $a \nless s b t$.

Proof. Assume that $I$ is an interior base of $S$. Then, $S=(I)_{I}$. Next, we need to show that (i) holds. Let $x \in S$. Thus, $x \in(I)_{I}=I \cup I I \cup S I S$. Now, we will consider three cases:

Case 1: $x \in I$. Then, there exists $a \in I$ such that $x=a$. This means that $(x)_{I}=(a)_{I}$, that is, $x \leqslant a$.

Case 2: $x \in I I$. Then, $x=a_{1} a_{2}$ for some $a_{1}, a_{2} \in I$. This implies that $(x)_{I}=\left(a_{1} a_{2}\right)_{I}$. Hence, $x \leqslant a_{1} a_{2}$. Case 3: $x \in S I S$. Then, $x=s a_{3} t$ for some $a_{3} \in I$ and $s, t \in S$. It turns out that $(x)_{I}=\left(s a_{3} t\right)_{I}$. So, $x \leqslant s a_{3} t$.

The conditions of (ii) and (iii) hold from Lemma 2.10(i) and Lemma 2.10(ii), respectively.

Conversely, assume that the conditions (i), (ii) and (iii) hold. We will show that $I$ is an interior base of $S$. Obviously, $(I)_{I} \subseteq S$. By (i), we have that for every $x \in S$, there exists $a \in I$ such that $x \leqslant a$. This implies that $x \in(x)_{I} \subseteq(a)_{I}=a \cup a a \cup S a S \subseteq$ $I \cup I I \cup S I S=(I)_{I}$. Hence, $S \subseteq(I)_{I}$. It follows that $S=(I)_{I}$. Next, we show that $I$ is a minimal subset of $S$ such that $S=(I)_{I}$. Suppose that $S=$ $(A)_{I}$ for some $A \subset I$. Then, there exists $x \in I \backslash A$. Since $x \in I \subseteq S=(A)_{I}$ and $x \notin A$, we have that $x \in A A \cup S A S$, Thus, we will consider two cases:

Case 1: $x \in A A$. Then, there exist $a_{1}, a_{2} \in A$ such that $x=a_{1} a_{2}$. We obtain that $a_{1}, a_{1} \in I$. Since $x \notin A, x \neq a_{1}$ and $x \neq a_{2}$. Since $x=a_{1} a_{2},(x)_{I} \subseteq$ $\left(a_{1} a_{1}\right)_{I}$. This implies that $x \leqslant a_{1} a_{2}$. This contradicts to the condition (ii).

Case 2: $x \in S A S$, Then, $x=s a_{3} t$ for some $a_{3} \in A$ and $s, t \in S$. Thus, $a_{3} \in I$ because $A \subset I$. Since $x \notin A, x \neq a_{3}$. Since $x=s a_{3} t,(x)_{I} \subseteq\left(s a_{3} t\right)_{I}$. This means that $x \leqslant s a_{3} t$, which is a contradiction to the condition (iii).

Therefore, $I$ is an interior base of $S$, This completes the proof.

In Example 2.2, we have that $\{c, d\}$ is an interior base of $S$, but it is not a subsemigroup of $S$. Hence, we also need find a requirement for an interior base to be a subsemigroup.

Theorem 2.12. Let I be an interior base of a semigroup $S$. Then I is a subsemigroup of $S$ if and only if I satisfies the following conditions: for any $b, c \in I$, $b c=b$ or $b c=c$.

Proof. Assume that $I$ is a subsemigroup of $S$. Let $b, c \in I$. Then, $b c \in I$. So, $b c \in b c \cup S b c S$. By Lemma 2.5, it follows that $b c=b$ or $b c=c$. The opposite direction is clear.

\section{Conclusion}

This paper deals with the concept of interior bases of a semigroup $S$ which obtained from interior ide- 
als generated by a nonempty subset of the semigroup $S$. Then, we investigated the quasi-order defined by the principal interior ideals of a semigroup $S$. Finally, we discussed a characterization of interior bases when a nonempty subset of $S$ is an interior ideal of $S$ (Theorem 2.11). Moreover, we have found the condition necessary for an interior base to be a subsemigroup (Theorem 2.12). It is known that every semigroup can be considered to be a semihypergroup. In our future study, we will extend the concept of interior bases to investigate in the structure of semihypergroups.

\section{Acknowledgment}

This research project is financially supported by

Mahasarakham University.

\section{References:}

[1] T. Tamura, One-sided bases and translations of a semigroup, Math. Japan., Vol. 3, 1995, pp. 137141.

[2] I. Fabrici, Two-sided bases of semigroups, Matematický časopis, Vol. 3, 2009, pp. 181-188.

[3] I. Fabrici and T. Kepka, One bases and maximal ideals in semigroups, Math. Slovaca, Vol. 31, 1981, pp. 115-120.

[4] T. Changphas and P. Sammaprab, On two-sided bases of an ordered semigroup, Quasigroups ans Related Systems, Vol. 22, 2014, pp. 59-66.

[5] P. Kummoon and T. Changphas, On bi-bases of a semigroup, Quasigroups ans Related Systems, Vol. 25, 2017, pp. 87-94.

[6] W. Jantanan, C. Budpan and W. Loesna, On ordered $\Gamma$-semihypergroups containing two-sided bases, Naresuan University Journal: Science and Technology (NUJST), Vol. 29, No. 3, 2021, pp. 96-104.

[7] A. Udom, T. Chokchai and S. Hobanthad, On bibases of semihypergroups, Mathematical Journal by The Mathematical Association of Thailand under the Patronage of His Majesty the King, Vol. 66, No. 703, 2021, pp. 14-31.

[8] W. Jantanan, P. Saiwan and N. Sankaew, On bibases of ordered $\Gamma$-semihypergroups, Journal of Science and Technology, Ubon Ratchathani University, Vol. 23, No. 1, 2021, pp. 12-20.

[9] S. Lajos, $(m, k, n)$-ideals in semigroups: notes on semigroups II, Karl Marx Univ. Econ., Dept. Math. Budapest, Vol. 1, 1976, pp. 12-19.

[10] Y. B. Jun and S. Z. Song, Generalized fuzzy interior ideals in semigroups, Information Sciences, Vol. 176, No. 20, 2006, pp. 3079-3093.

[11] N. Kehayapulu, Note on interior ideals, ideal elements in ordered semigroups, Scientiae Mathematicae, Vol. 2, No. 3, 1999, pp. 407-409.

[12] A. Khan, Y. B. Jun and T. Mahmood, Generalized fuzzy interior ideals in Abel Grassmann's groupoids, International Journal of Mathematics and Mathematical Sciences, Vol. 2020, 2020, pp. $1-14$.

[13] K. H. Kim and Y. B. Jun, Intuitionistic fuzzy interior ideals of semigroups, International Journal of Mathematics and Mathematical Sciences, Vol. 25, No. 7, 2001, pp. 261-267.

[14] D. Mandal, Fuzzy ideals and fuzzy interior ideals in ordered semirings, Fuzzy Information and Engineering, Vol. 6, No, 1, 2014, pp. 101-114.

[15] S. K. Sardara, B. Davvaz and S. K. Majumder, A study on fuzzy interior ideals of $\Gamma$-semigroups, Computers \& Mathematics with Applications, Vol. 60, No. 1, 2010, pp. 90-94.

Creative Commons Attribution License 4.0 (Attribution 4.0 International, CC BY 4.0) This article is published under the terms of the Creative Commons Attribution License 4.0 https://creativecommons.org/licenses/by/4.0/deed.en US 